\title{
Erratum to: A multidisciplinary approach to enhance the conservation and use of hazelnut Corylus avellana $\mathbf{L}$. genetic resources
}

\author{
L. Bacchetta - M. Rovira - C. Tronci - M. Aramini - \\ P. Drogoudi - A. P. Silva - A. Solar - D. Avanzato • \\ R. Botta $\cdot$ N. Valentini $\cdot$ P. Boccacci
}

Received: 1 January 2014/ Accepted: 27 August 2014/Published online: 9 May 2015

(C) Springer Science+Business Media Dordrecht 2015

\section{Erratum to: Genet Resour Crop Evol \\ DOI 10.1007/s10722-014-0173-7}

We regret that the table part of Fig. 3 was missed in the online publication. The correct figure is given below.
The online version of the original article can be found under doi:10.1007/s10722-014-0173-7.

L. Bacchetta $(\bowtie) \cdot$ C. Tronci · M. Aramini

Agenzia Nazionale per le Nuove Tecnologie, l'Energia e lo Sviluppo Economico Sostenibile, ENEA Casaccia UTAGRI, Via Anguillarese, 301, 000123 Rome, Italy e-mail: loretta.bacchetta@enea.it

\section{Rovira}

Division IRTA, Institut de Recerca i Tecnologia Agroalimentàries, Centre Mas de Bover, Ctra. Reus-El Morell, km 3.8, 43120 Constantí, Tarragona, Spain

P. Drogoudi

Pomology Institute, Hellenic Agricultural Organization 'Demeter', D.G. of Agricultural Research, 38 R.R.

Station, 59035 Naoussa, Greece

A. P. Silva

CITAB, Centre for the Research and Technology of Agro-

Environment and Biological Sciences, Universidade de

Trás-os-Montes e Alto Douro, Apartado 1013,

5001-801 Vila Real, Portugal

\author{
A. Solar \\ Biotehniska Fakulteta, Univerza v Ljubljani, Jamnikarjeva \\ 101, 1000 Ljubljana, Slovenia \\ D. Avanzato \\ CRA Centro Ricerca per la Frutticoltura, Via Fioranello, \\ 52, 0013 Rome, Italy \\ R. Botta $\cdot$ N. Valentini \\ Department of Agricultural, Forestry and Food Sciences \\ (DISAFA), University of Turin, Largo Paolo Braccini, 2, \\ 10095 Grugliasco, TO, Italy \\ P. Boccacci \\ Institute for Sustainable Plant Protection - National \\ Research Council (IPSP-CNR), UOS of Grugliasco, Largo \\ Paolo Braccini, 2, 10095 Grugliasco, TO, Italy
}




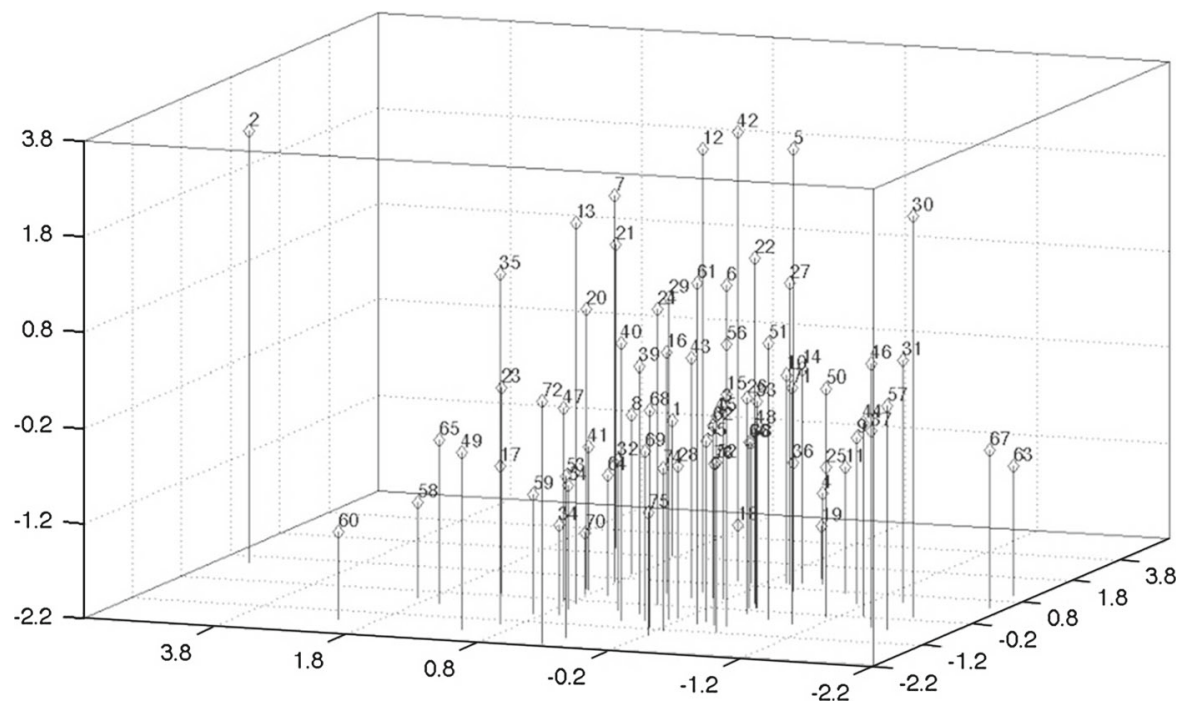

\begin{tabular}{llll}
\hline Variable & PC1 & PC2 & PC3 \\
\hline $\begin{array}{l}\text { oil content } \\
\%\end{array}$ & $\mathbf{- 0 . 5 1 4}$ & -0.329 & -0.259 \\
C16:0 & -0.302 & $\mathbf{0 . 8 3 3}$ & -0.102 \\
C16:1 & -0.16 & 0.597 & -0.433 \\
C18:0 & -0.68 & 0.381 & 0.24 \\
C18:1 & $\mathbf{- 0 . 7 3 7}$ & -0.649 & 0.068 \\
C18:2 & $\mathbf{0 . 9 1 8}$ & 0.343 & -0.095 \\
C18:3 & 0.485 & 0.178 & 0.185 \\
SFA & -0.585 & $\mathbf{0 . 8 0 0}$ & 0.058 \\
Total & $\mathbf{- 0 . 7 4 2}$ & -0.642 & 0.062 \\
MUFA & & & \\
Total PUFA & $\mathbf{0 . 9 2}$ & 0.343 & -0.09 \\
UFA/SFA & 0.625 & -0.765 & -0.065 \\
MUFA/SFA & 0.362 & -0.913 & -0.03 \\
PUFA/SFA & 0.98 & 0.041 & -0.125 \\
alfa- & 0.258 & -0.084 & $\mathbf{0 . 9 1}$ \\
tocopherol & & & \\
SI & 0.087 & 0.259 & $\mathbf{0 . 9 2 2}$ \\
Eigenvalue & 5.759 & 4.538 & 2.085 \\
Proportion & 38.395 & 30.257 & 13.897 \\
of total (\%) & & & \\
\hline
\end{tabular}

Fig. 3 Position of the principal component (PC) scores of the hazelnut kernel composition for 75 hazelnut cultivars. Numbers refer to the clone number of the cultivar: 1 C.a. 9 Raul; 2 Gunslebert; 3 Barcellona; 4 Hall's Giant; 5 Segorbe; 6 Dal Rosso; 7 C. maxima à Pellicule Blanche; 8 Negret; 9 Fertile de Coutard; 10 Bergeri; $11 \mathrm{CV} / 2$; 12 Rotblattrige Lambertnuss; $13 \mathrm{C}$. maxima à Pellicule Rose; 14 Merveille de Bollwiller; 15 Longue d'Espagne; 16 Pauetet (ref. cv.); 17 Incrocio L35; 18 Trenet; 19 Vermellet; 20 Corabel; 21 Casina; 22 Nostrale; 23 Provence; 24 Pallagrossa; 25 CV/1; 26 Tonda di Giffoni (ref. cv.); 27 Pellicola bianca; 28 San Giovanni; 29 Tonda bianca; 30 Ferwiller, 31 Istrska okrogloplodna leska; 32 Tonda Gentile Langhe (ref. cv.);
33 Feriale; 34 Polycarpos wild; 35 San Vicino; 36 Molar; 37 Lunga Ginnasi; 38 Gironell; 39 Tonda di Biglini; 40 TGL d(clone PD); 41 C.a. 5 Grada de Viseu, 42 Cosford; 43 C.a. 11 Tubulosa; 44 Istrska dolgoplodna leska; 45 Nocchione; 46 Imperatrice Eugenie; 47 C.a. 12 Purpurea; 48 C.a. 7 DaVeiga; 49 GR pi 03; 50 Piazza armerina; 51 Nociara; 52 Riccia di Talanico; 53 Meloni; 54 Karydato; 55 Carrello; 56 Tonda gentile romana; 57 Avellana Speciale; 58 Argiroupoli; 59 Patem; 60 Palaz; 61 Ada; 62 Barettona Vico; 63 C.a. 8 Comum; 64 Castanyera (ref. cv.); 65 Extra Giaghli; 66 Morell; 67 Grifoll; 68 Camponica; 69 Daria; Tombul Giaghli; 71 Comune di Sicilia; 72 Tonda CalabreseCaserta; 73 Barettona; 74 Centenaria Ginnasi; 75 Culpla 